\title{
Near-IR spectroscopic search for binary companions to dusty WC stars
}

\author{
Watson P. Varricatt \\ Joint Astronomy Centre, Hawai'i Headquarters, University Park, \\ 660 North A'ohoku Place, Hilo, HI96720, USA
}

\begin{abstract}
Many late WC type Wolf-Rayet (WR) stars are known to form dust in their winds. While, in the case of episodic dust makers, it is now clear that dust formation takes place due to a colliding wind phenomenon, we still do not know for sure if binarity is the main reason for dust formation in persistent dust makers. This work is to understand, if persistent dust formation is also due to colliding winds in close binary systems. A systematic search for colliding winds is taken up via high resolution spectroscopy of the He I $\lambda 1.083 \mu \mathrm{m}$ emission line. Preliminary indications of binarity in many persistent dust makers are seen.
\end{abstract}

\section{Introduction}

Based on the nature of IR variability due to dust formation, dusty WC stars are classified as episodic and persistent dust makers (Williams 1995). Episodic dust makers, which show periodic infrared brightning, are now understood to be $\mathrm{WC}+\mathrm{O}$ binaries in elliptical orbits where, during epochs close to periastron passage, a colliding wind phenomenon triggers dust formation. Persistent dust makers are known to form dust around them always. The purpose of this observational study is to understand the nature of persistent dust making WC stars, i.e., are they close binaries or there is some other mechanism responsible for dust formation?

\section{Observations and discussions}

Observations were done with the UKIRT-CGS4, using an echelle grating with a spectral resolution of $R=18500$, using a 2 pixel slit. To increase the $\mathrm{S} / \mathrm{N}$, the spectra presented were binned in 5 pixels. Line emission from the colliding wind region can be seen as a separate emission component superposed on the broad WR emission line (Lührs 1995, Stevens \& Howarth 1999). The flat topped He I $\lambda 1.083 \mu \mathrm{m}$ emission line, which is very strong in late WC stars, is a very good tracer of colliding winds in WR+O binaries. This line is formed at the outskirts of WR winds, where the wind has already attained its terminal velocity.

Most of the stars observed show very clear sub-humps (WR 98a, WR 104, WR 112 and WR 119). WR 98a is an episodic dust maker (Williams 1995). Monnier, Tuthill \& Danchi (1999) discovered a 'pinwheel' dust shell nebula around this object, confirming the binarity. The period of the binary system is $565 \pm 50 \mathrm{~d}$. A broad and well defined subhump is seen on the He I line of 

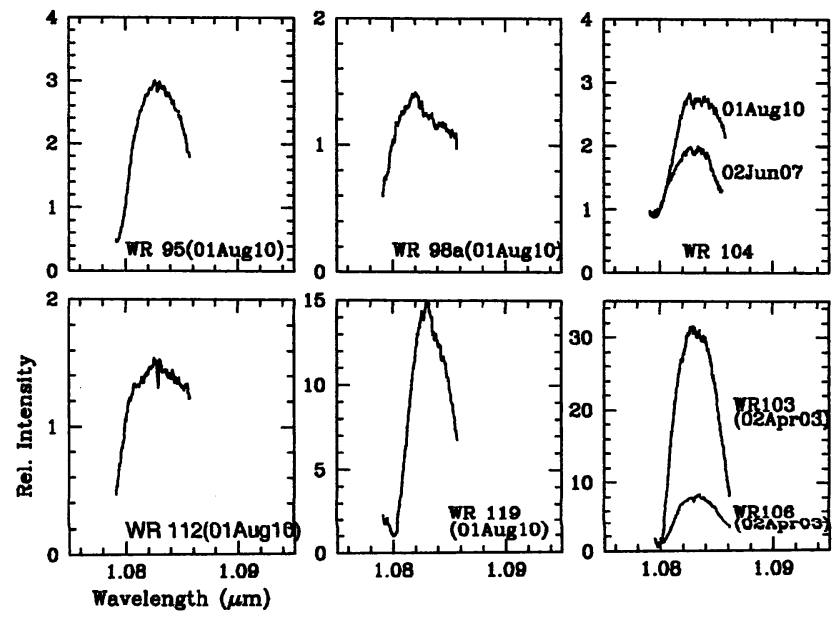

Figure 1. Echelle spectra of the $\mathrm{HeI} \lambda 1.083 \mu \mathrm{m}$ line.

this star. WR 104, which also is a persistent dust making binary with a dusty pinwheel nebula (Tuthill, Monnier \& Danchi 1999), shows faint subpeaks. Two epochs of observation of this star shows large variations in the line flux. WR 112 and WR 119 also show well defined subpeaks. Recent observations of WR 112 also show pinwheel type of dust shell around it (Marchenko et al. 2002). WR 95, WR 103 and WR 106 also show slight line profile assymmetries which will be monitored.

Out of $\sim 27$ Galactic WC9 stars known (van der Hucht 2001), except a few, most of them are dust makers, either episodic or persistent, more being persistent. So, studying the binary nature of dust makers can make a significant impact on our understanding of evolution leading to WC9 phase and the nature of WC9 stars.

Acknowledgments. UKIRT is operated by the Joint Astronomy Centre, Hilo, Hawaii on behalf of the UK Particle Physics and Astronomy Research Council. I thank Andrew J. Adamson for obtaining the spectrum of WR 104.

\section{References}

van der Hucht, K.A. 2001, New Astron. Reviews 45, 135

Lührs, S. 1995, in: K. A. van der Hucht \& P. M. Williams (eds.), Wolf Rayet Stars: Binaries, Colliding Winds, Evolution, Proc. IAU Symp. No. 163 (Dordrecht: Kluwer), p. 416

Marchenko, S.V., Moffat, A.F.J., Vacca, W.D., Cote, S., Doyon, R. 2002, ApJ (Letters) $565, \mathrm{~L} 59$

Monnier, J.D., Tuthill, P.G., Danchi, W.C. 1999, ApJ (Letters) 525, L97

Stevens, I.R., Howarth, I.D. 1999, MNRAS 302, 549

Tuthill, P.G., Monnier, J.D., Danchi, W.C. 1999, Nature 398, 487

Williams, P.M. 1995, in: K.A. van der Hucht \& P.M. Williams (eds.), Wolf Rayet Stars: Binaries, Colliding Winds, Evolution, Proc. IAU Symp. No. 163 (Dordrecht: Kluwer), 335 